\title{
Neutrino Oscillations in a Minimal CPT Violation Frame
}

\author{
Paola Arias* and J. Gambod \\ Departamento de Física, Universidad de Santiago de Chile, Casilla 307, Santiago, Chile
}

\begin{abstract}
The oscillation neutrino problem in the extended standard model with minimal number of parameters is considered. The dispersion relations with explicit neutrino-antineutrino asymmetry are discussed and an explanation to the MINIBooNE and solar neutrino controversy is offered. Bounds for the CPT violation symmetry are also found.
\end{abstract}

\section{INTRODUCTION}

The analysis and design of neutrino oscillation experiments have been a very active research field due possible evidence of CPT violation in neutrino physics. Since the controversial LSND result [1], experiments such as KARMEN [2] and specially MINIBooNE [3, 4] have been designed to test the LSND results, which seem to indicate a signal of $\mathrm{CPT}$ violation in neutrino oscillation factories.

As well known, the LSND experiment explored the transition $\bar{\nu}_{\mu} \rightarrow \bar{\nu}_{e}$ and providing a positive appearance of $\bar{\nu}_{e}$ over the background. This last fact suggest neutrino oscillations at short baseline, with a best fit in $\Delta m_{\text {LSND }}^{2} \sim 1$ $\mathrm{eV}^{2}$.

Clearly -if this result is true and the CPT theorem holds- then this is in contradiction with solar neutrino data where the transition $\nu_{e} \rightarrow \nu_{\mu}$ is favored with a best fit of $\Delta m_{S O L}^{2} \sim 5.5 \times 10^{-5} \mathrm{eV}^{2}$ and, therefore, the LSND results could require an explanation beyond of conventional physics.

The MiniBooNE experiment was specially designed to test the LSND results and, in a first phase, the channel $\nu_{\mu} \rightarrow \nu_{e}$ was tested by exploring energy ranges between $(400-3000) \mathrm{MeV}$. No significant result over the background was reported to high energies and LSND allowed region was excluded with a 90\%CL. However, a slightly appearance of $\nu_{e}$ at low energies was detected [5].

Motivated by this results, MiniBooNE repeated their measurements, extending the low energy range to $200 \mathrm{MeV}$. The excess of $\nu_{e}$ events was confirmed indicating a positive sign of oscillation, with an enhancement in the $300 \mathrm{MeV}$ range. The best fit in the low energy regime is for $E>200 \mathrm{MeV}$ and $\Delta m^{2}=3.14 \mathrm{eV}^{2}$ and $\sin ^{2} 2 \theta=1.7 \times 10^{-3}$. Although this result is incompatible with LSND-type oscillations, has prompted several research activity because put us back where we were: incompatibility between solar and accelerator experiments. In addition to the above, presently MiniBooNE is running the experiment in the antineutrino mode, $\bar{\nu}_{\mu} \rightarrow \bar{\nu}_{e}$, under same baseline conditions and energy ranges. Surprisingly, no excess of $\bar{\nu}_{e}$ events have been reported for low energy (nor high) range [6].

The MiniBooNE result open the interesting possibility [7, 8] of consider a tiny CPT violation in order to explain, in one hand the absence of oscillation in the antineutrino mode and, on the other hand, the $\Delta m_{M B}^{2} \sim \mathrm{eV}^{2}$ which is in contradiction with solar neutrino reports.

In this paper we would like to explore a minimal CPT violating model [9], based on the extended standard model $(\mathrm{ESM})[10,11,12]$. Basically in this model we take the neutrino ESM sector and then the corresponding dispersion relations are obtained. These dispersion relations have explicit neutrino-antineutrino asymmetry and this fact allows, in one hand, to reconcile the MiniBoone and solar data and on the other hand, to get bounds for the CPT violating parameters for this experiments. Other bounds for CPT violation symmetry in different neutrino oscillation experiments are also computed and discussed.

\section{CPT VIOLATION LAGRANGIAN AND EQUATIONS OF MOTION}

Our starting point is a minimal subset of the SME Lagrangean [10, 11]

$$
\mathcal{L}=\bar{\nu}_{a}\left(i \Gamma_{a b}^{\mu} \partial_{\mu}-m_{a b}\right) \nu_{b},
$$

\footnotetext{
*Electronic address: paola.arias@gmail.com
}

†Electronic address: jgamboa55@gmail.com 
with $a, b$ flavor indices and

$$
\Gamma_{a b}^{\mu}=\gamma^{\mu} \delta_{a b}+e_{a b}^{\mu} .
$$

$m_{a b}$ is the standard CPT conserving mass matrix and we will assume two-neutrino mixing.

In order to preserve rotational invariance, we chose $e_{a b}^{\mu} \rightarrow e_{a b}^{0}$ and, therefore, the equations of motion -written in momentum space- becomes

$$
\left[(E-\vec{\alpha} \cdot \vec{p}) \delta_{a b}-\left(m_{a b}-E e_{a b}\right) \gamma^{0}\right] \nu_{b}=0 .
$$

If we choose the chiral representation for the gamma matrices one find that (3) yield to the following dispersion relations

$$
\begin{aligned}
& E_{ \pm}^{(1)}=-\frac{m_{1} e_{1}}{1-e_{1}^{2}} \pm \frac{1}{\sqrt{1-e_{1}^{2}}} \sqrt{m_{1}^{2}+p^{2}+\frac{m_{1}^{2} e_{1}^{2}}{1-e_{1}^{2}}}, \\
& E_{ \pm}^{(2)}=-\frac{m_{2} e_{2}}{1-e_{2}^{2}} \pm \frac{1}{\sqrt{1-e_{2}^{2}}} \sqrt{m_{2}^{2}+p^{2}+\frac{m_{2}^{2} e_{2}^{2}}{1-e_{2}^{2}}},
\end{aligned}
$$

where the \pm signs denote particle $(+)$ and antiparticle $(-)$ and $m_{1,2}$ and $e_{1,2}$ correspond to the eigenvalue of matrix $m$ and $e$, respectively. Note that (4) and (5) have particle-antiparticle asymmetry and, therefore, explicit CPT violation.

The mixing angle is fixed to Large Mixing approximation (LMA) because the elements off diagonal in $m$ and $e$ matrices were set to zero. However, if we consider a more general set of matrices $m$ and $e$ with the same basis of eigenvectors, then the mixing angle would be not fixed and has an energy dependence, with different values for neutrino and antineutrinos.

Next step is to expand the above dispersion relations in powers of $m^{2} / p^{2}$, thus

$$
E_{a}^{ \pm}=\mp m_{a} e_{a}+p\left(1+\frac{m_{a}^{2}}{2 p^{2}}+\frac{e_{a}^{2}}{2}\right)
$$

where $p\left(1+m^{2} / p^{2}\right)$ correspond to the first terms of the expansion $\sqrt{p^{2}+m^{2}}$ and the following ones are Lorentz and CPT violating contributions (see e.g. $[13,14]$ ). We finalize this section by noticing that the CPT violation have been considered only at the kinetic level and this fact is enough to generate (6).

\section{OSCILLATION PROBABILITY IN CPT VIOLATION FRAME}

Recall that the probability of oscillation between two different species of neutrinos in a CPT conserving scenario, say $\nu_{\alpha}$ and $\nu_{\beta}$ is given by

$$
P_{\nu_{\alpha} \rightarrow \nu_{\beta}}=\sin ^{2} 2 \theta \sin ^{2}\left(\frac{L}{L_{0}}\right)
$$

where $L$ is the length from the production source to the detector and $L_{0}$ is the so-called oscillation length, given by

$$
L_{0}=\frac{4 \pi E}{\Delta m_{i j}^{2}}
$$

In order to observe oscillations, it must be satisfied that $L / L_{0} \geq 1$.

Maximal sensitivity to oscillations, and therefore $\Delta m_{i j}^{2}$, is obtained when the setup of the experiment is such that

$$
\frac{E}{L} \approx \Delta m_{i j}^{2} .
$$


In our CPT violation scenario, the oscillation probability between two different neutrino species is computed to be

$$
P_{\nu_{\alpha} \rightarrow \nu_{\beta}}=\sin ^{2} 2 \theta \sin ^{2}\left(\Delta E_{a b} L\right)
$$

where $\Delta E_{a b}=E_{a}-E_{b}$. Thus, using the dispersion relation, eq. (6) we obtain

$$
P_{\nu_{\alpha} \rightarrow \nu_{\beta}}=\sin ^{2} 2 \theta \sin ^{2}\left(\frac{\Delta m_{12}^{2}}{2 E} L+\frac{\Delta e_{12}^{2} E}{2} L+\left(m_{2} e_{2}-m_{1} e_{1}\right) L\right)
$$

and for antineutrinos

$$
P_{\bar{\nu}_{\alpha} \rightarrow \bar{\nu}_{\beta}}=\sin ^{2} 2 \bar{\theta} \sin ^{2}\left(\frac{\Delta m_{12}^{2}}{2 E} L+\frac{\Delta e_{12}^{2} E}{2} L-\left(m_{2} e_{2}-m_{1} e_{1}\right) L\right) .
$$

The bar over the mixing angle accounts for the possibility of consider a different mixing angle for particle and antiparticle, as was discussed before.

The first term in sine argument corresponds to the standard difference of squared masses over energy. The rest corresponds to the contribution of CPT violation.

The term independent of the energy, of the form $\eta_{a b}^{0}=m_{a} e_{a}-m_{b} e_{b}$, has been constrained in [13] using bounds for atmospheric neutrinos to $\eta_{23}^{0}<10^{-23} \mathrm{GeV}$. Meanwhile, from solar neutrino data [14] the corresponding bound is $\eta_{12}^{0}<10^{-21} \mathrm{GeV}$. As expected, this term is well attenuated, and has almost none influence in the oscillation phenomena.

The oscillation length is modified, adding a term which becomes significant at high energies

$$
L_{0}^{C P T V}=\frac{1}{\frac{\Delta m^{2}}{4 \pi E}+\frac{\Delta e^{2} E}{2}} .
$$

Neutrino transitions can be observed if $L \geq L_{0}^{C P T V}$ and, therefore, one has

$$
\Delta m_{i j}^{2} X\left(1+\frac{\Delta e_{i j}^{2} E^{2}}{\Delta m_{i j}^{2}}\right) \geq 1
$$

where $X=L / E$. Experimental setups are arranged such that $\Delta m_{i j}^{2} X \sim 1$. Using this expression we can find bounds for $\Delta e_{i j}^{2}$ from different experimental data.

\section{A. Bounds on $\Delta e_{i j}^{2}$}

According to the MiniBooNE setup, the distance from the source to the detector is $L=541 \mathrm{~m}$ and the range of energy for observed oscillations is $300-475 \mathrm{MeV}$, with $\Delta m_{12}^{2}$ of the order of $1 \mathrm{eV}^{2}$. Using formulae (14) we can find a bound of $\Delta e_{12}^{2}$

$$
\Delta e_{12}^{2} \leq 10^{-18}, \quad \text { for } \quad \Delta m_{12}^{2} \sim 1 \mathrm{eV}^{2}
$$

Using this bound for $\Delta e_{12}^{2}$, is clear that for solar neutrino oscillations the CPT violation is well suppressed. Indeed, the solar neutrino data are $E=0.8 \mathrm{MeV}$ and $\Delta m_{12}^{2} \sim 10^{-5}$ and, therefore the coefficient must satisfy

$$
\frac{\Delta e_{12}^{2} E^{2}}{\Delta m_{12}^{2}}<<1
$$

The absence of neutrino oscillations in MiniBooNE for the range of energies over $500 \mathrm{MeV}$ is very natural; the coefficient $X<<1$ and besides, $\frac{\Delta e_{i j}^{2} E^{2}}{\Delta m_{i j}^{2}} \leq 1$ in the range of energy between $500-3000 \mathrm{MeV}$. Thus, the oscillation is 
suppressed.

If we try to conciliate the solar and MiniBooNE masses, one should set $\Delta m_{12}^{2}=\Delta m_{S O L}^{2}=\Delta m_{M B}^{2} \sim 10^{-5} \mathrm{eV}^{2}$, in which case the bound for $\Delta e_{12}^{2}$, using the above formula, becomes

$$
\left|\Delta e_{12}^{2}\right| \leq 10^{-17} .
$$

Using atmospheric neutrino oscillation data we can constraint the parameter $\Delta e_{23}^{2}$. Indeed, using existing data for $\Delta m_{23}^{2} \sim 10^{-3} \mathrm{eV}^{2}, X \sim 10^{-3} \mathrm{eV}^{2}$, and the neutrino energy in multi-GeV events $E \sim 3 \mathrm{GeV}$, we obtain the bound

$$
\Delta e_{23}^{2} \leq 10^{-21}
$$

\section{CONCLUSIONS}

In this paper we have proposed a minimal version of the ESM containing two free parameters responsible of the CPT violation in the neutrino sector. In connection with the oscillation probability, we found that the oscillation length is modified by the inclusion of the CPT violation parameters (see also [15]) with a minimal number of free parameters. Thus, the simplified model studied here allowed us to constraint the parameters that determines the violation of symmetry.

From the MiniBooNE results for low-energy neutrino oscillation we find the bound $\Delta e_{12}^{2}=e_{1}^{2}-e_{2}^{2} \leq 10^{-18}$, however an agreement between MiniBooNE and solar neutrino data can be reached if $\left|\Delta e_{12}^{2}\right|$ is one order of magnitude below i.e. $\Delta e_{12}^{2} \leq 10^{-17}$. For completeness, the bound for $\Delta e_{23}^{2}$ was also obtained, using the atmospheric neutrino oscillation data. The bound is $\Delta e_{23}^{2} \leq 10^{-21}$.

It is interesting to note that from the dispersion relation obtained in the paper a possible scenario of neutrinoantineutrino oscillation also could be considered. For example, the difference $\left|E_{a}^{+}-E_{a}^{-}\right| \approx 2 m_{a} e_{a}$ tell us that, if the mixing angle is not suppressed, then an oscillation could appear as a CPT violation effect [16] (the same conclusion would be reached by considering an oscillation between neutrino-antineutrino of different species).

Acknowledgements: We would like to thank J. López-Sarrión by fruitful discussions. This work was partially supported by FONDECYT-Chile grant-1095106 and DICYT (USACH).

[1] C. Athanassopoulos et al., Phys. Rev. Lett. 75, 2650 (1995); 77, 3082 (1996); 81, 1774 ( 1998); A. Aguilar et al., Phys. Rev. 64, 112007 (2001).

[2] K. Eitel et al. [KARMEN collaboration], Nucl. Phys. Proc. Suppl. 77, 212 (1999).

[3] A. Aguilar-Arevalo et al., Phys. Rev. Lett. 98, 231801 (2007).

[4] A. Aguilar-Arevalo et al., Phys. Rev. D78,012007 (2008).

[5] A. A. Aguilar-Arevalo et al. [MiniBooNE Collaboration], Phys. Rev. Lett. 102, 101802 (2009).

[6] A. A. Aguilar-Arevalo et al., arXiv:0904.1958 [hep-ex].

[7] C. Giunti and M. Laveder, "VSBL Electron Neutrino Disappearance," arXiv:0902.1992 [hep-ph].

[8] C. Giunti and M. Laveder, Phys. Rev. D 77, 093002 (2008).

[9] S. Coleman and S. L. Glashow, Phys. Lett. B405, 249 (1997); ibid, Phys. Rev. D59, 116008 (1999); P. Arias et al, Phys. Lett. B650, 401 (2007); J. Carmona, et al, JHEP 0806, 033 (2008).

[10] V. A. Kostelecky and M. Mewes, Phys. Rev. D 69, 016005 (2004).

[11] V. A. Kostelecky and M. Mewes, Phys. Rev. D 70, 031902 (2004).

[12] D. Colladay and V. A. Kostelecky, Phys. Rev. D58, 116002 (1998).

[13] E. Lisi, A. Marrone, D. Montanino, Phys. Rev. Lett. 85, 1166 (2000).

[14] J. N. Bahcall, V. Barger, D. Marfatia, Phys. Lett. B534, 120 (2002).

[15] G. L. Fogli, E. Lisi, A. Marrone and G. Scioscia, Phys. Rev. D 60, 053006 (1999).

[16] For a discussion along these lines see e.g., S. Hollenberg, O. Micu and H. Pas, "Neutrino-antineutrino oscillations as a possible solution for the LSND and MiniBooNE anomalies?," arXiv:0906.5072 [hep-ph]. 